\title{
Hypothetical Flora and Fauna on the Planet Venus Found by Revision of the TV Experiment Data (1975-1982)
}

\section{Leonid Ksanfomality, Arnold Selivanov, Yuryi Gektin}

Space Research Institute of the Russian Academy of Sciences, Moscow, Russia

Email address:

leksanf@gmail.com (L. Ksanfomality)

\section{To cite this article:}

Leonid Ksanfomality, Arnold Selivanov, Yuryi Gektin. Hypothetical Flora and Fauna on the Planet Venus Found by Revision of the TV Experiment Data (1975-1982). American Journal of Modern Physics. Vol. 7, No. 1, 2018, pp. 34-47. doi: 10.11648/j.ajmp.20180701.15

Received: October 11, 2017; Accepted: November 13, 2017; Published: January 11, 2018

\begin{abstract}
We present the results of new processing the archival telemetry data, returned from the surface of Venus by the VENERA spacecraft landers in 1975 and 1982. Reprocessing of the unique data with up-to-date techniques resulted in substantial improvement of the level of details. New analysis of the VENERA television images led to detection and identification of a dozen of hypothetical living-like objects. Many objects with a complex regular structure and presumably very slow motions (in the case of hypothetical fauna) have been found. These sizable objects may potentially indicate the existence of life on Venus with its radically different physical conditions. We call for a new special mission, much more sophisticated than the VENERA missions (1975-1982), should be urgently carried out to investigate the hypothetical life on Venus.
\end{abstract}

Keywords: VENERA Missions, Extraterrestrial Life, Hypothetical Venusian Flora and Fauna, Terramorphism

\section{Introduction}

Possibly, the answer to the question of the existence of extraterrestrial life may be found not on other worlds removed by distances of tens of parsecs, but on the surface of a nearest planet in the Solar system, on Venus. This conclusion follows from new processed results of the television experiments on the planet's surface performed by the VENERA landers in 1975 and 1982 [1-4]. The in-situ television (TV) investigations of the surface of Venus remain a unique experiment. In 1975, two probes of the VENERA-9 and -10 missions of the Soviet VENERA series landed on the surface of the planet. One of the main experiments was the pioneering TV experiment carried out for investigations of the surface of the planet. Seven years later, in 1982, more sophisticated TV experiments were performed in the course of the VENERA-13 and -14 missions.

Returning of TV images is a widely used up-to-date method for studying celestial bodies and searching for traces of life in space. Nowadays the planet Mars is being intensively investigated in this way. However, as far back as 40 years, the VENERA landers (Figure 1) investigated the surface of Venus with the TV method. Experiments onboard the VENERA probes were designed to gain general ideas about the surface of the planet. It did not occur to anybody at that time to search for life on a planet with an anoxic carbon dioxide atmosphere having a pressure of $9.2 \mathrm{MPa}$, and a temperature of $735 \mathrm{~K}$ near the surface. However, now it cannot be ruled out that the TV images of the surface of Venus, taken 42 and 35 years ago and reprocessed with uptodate software, evidence the presence of flora and fauna on this planet, strange as this may seem. Since experimental data on the supposed habitability of Venus were It is quite possible, that extraterrestrial life may be found not in other worldstens of parsecs afar, but on the surface of the closest to us planet in the Solar system, Venus. Such conclusion follows from recent processing of the television data of the planet's surface taken by the VENERA landers in 1975 and 1982 [1-4]. The in-situ television (TV) investigations of the surface of Venus remain unique. In 1975, two probes of the VENERA-9 and -10 missions of the Soviet VENERA series landed on the surface of Venus. One of the main experiments was the pioneering TV shooting to investigate the surface of the planet. Seven years later, in 1982, more sophisticated TV experiments were performed by VENERA-13 and -14 missions.

Transmission of TV images is a widely used up-to-date method for studying celestial bodies and searching for traces 
of life in space. E.g., the planet Mars is being intensively investigated in this way nowadays. However, as far as 40 years ago, VENERA landers (Figure 1) investigated the surface of Venus with the TV method. Experiments onboard of VENERA probes were designed to gain general information about the surface of the planet. It did not occur to anybody at that time to search for life on a planet with an anoxic carbon dioxide atmosphere having a pressure of 9.2 $\mathrm{MPa}$, and a temperature of $735 \mathrm{~K}$ near the surface. However, now it cannot be ruled out that the TV images of the surface of Venus, taken 42 and 35 years ago and reprocessed with uptodate software, indicate the presence of flora and fauna on this planet, strange as this may seem. Since experimental data on the supposed habitability of Venus were obtained for the first time, it was impossible to count on the support of specialists. However, what kind of life they looked for? obtained for the first time, it was impossible to count on the support of specialists. However, what kind of life they were looking for?

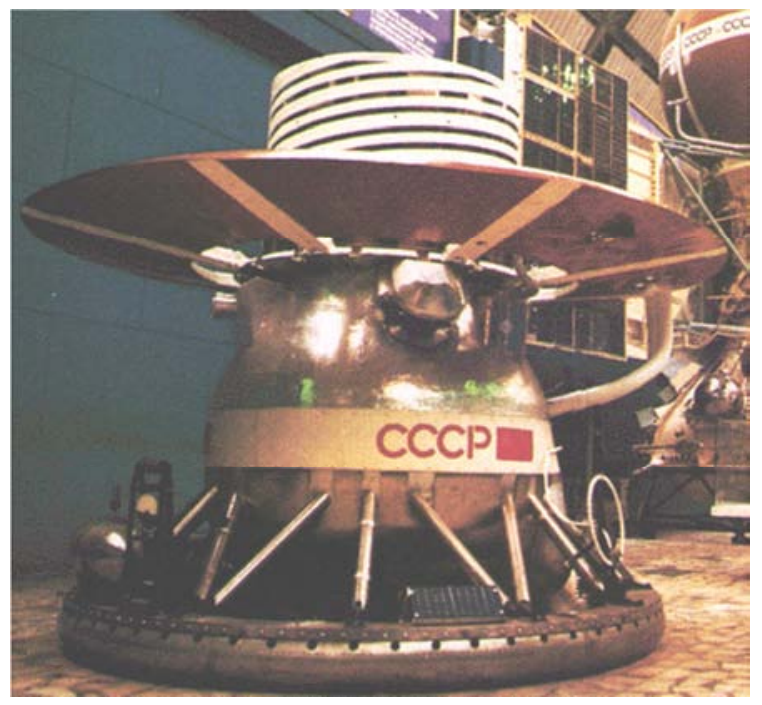

Figure 1. VENERA-13 lander at laboratory tests.

Looking for signs of life beyond the Earth, researchers usually proceed from the physical features that the Earth life possesses. In addition, it is assumed that the hypothetically detected life will not be too much different from ours. Due to intrinsic "Earthly chauvinism" it is also assumed that our physical settings are the best, too. Thus, in essence, in search for habitability we are looking for ourselves and it is obligatory to look for an atmosphere contains oxygen, as a sign of life, though experts argue that the first 3 billion years earthly life existed well without atmospheric oxygen. And yet - the surface temperature of a habitable planet should only be, as is comfortable for us. On the other hand, chemists remind us that at high temperature, reactions are accelerated considerably. Even compounds are produced which do not arise under normal conditions. And a significant part of exoplanets has high temperatures on their surface.

Nevertheless, many researchers believe that the search for life outside the Earth should be extended to completely different physical conditions. Well-known biochemist I.
Azimov, considered the chemistry of life existing at higher temperatures and called for nucleic acids and proteins based on nitrogen, rather than carbon. In the book "Life in the Solar System and Beyond," B. Jones writes (page 88): "Our approaches should not be built too literally on the variant of life that exists on Earth, i.e. based on RNA, DNA (carbon and liquid water) and a specific set of proteins... Following some search options, we would be able to detect life based on a completely different chemical composition (without carbon and water)." Leading Russian biophysicist A. Spirin, considering first reports on the discovery of a hypothetical life on Venus, at temperatures of $460^{\circ} \mathrm{C}$, noted: "Being a molecular biologist,... I have found nothing that would principally contradict the possibility that the living organisms exist under Venusian settings."

\section{TV-Experiment Onboard the Venera Landers}

There are discussions suggesting that the TV experiments should be described in more detail. Forty two years ago, the first TV images were received from the surface of Venus. At that time, Soviet investigations of the closest planet carried out with spaceborne instruments were at their height. The VENERA probes performed complex experiments when descending in the atmosphere of the planet. The atmosphere of Venus is so dense that the first module probing the atmosphere, VENERA-4 (1967) was crashed at a pressure of $0.72 \mathrm{MPa}$ at an altitude of around $25 \mathrm{~km}$ above the surface. The next, VENERA-5 and -6 probes (1969) experienced a pressure of $2.7 \mathrm{MPa}$ measured at an altitude of approximately $17 \mathrm{~km}$ and were crushed by the atmosphere, too.

The VENERA-7 (1970) and VENERA-8 probes (1972) were the first to reach the surface in an operating state. The first surface images, "panoramas", were returned by the VENERA-9 and -10 spacecraft in fall 1975. Under the atmospheric pressure exceeding Earth's by 92 times and at a temperature of $460^{\circ} \mathrm{C}$, they operated for almost an hour before they were destroyed. Both of landers VENERA-9 VENERA-10 were equipped with special TV cameras [3].

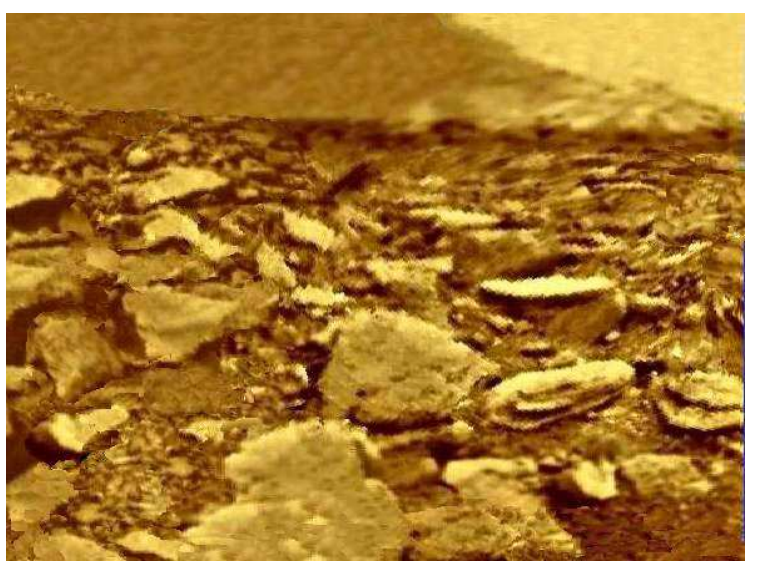

Figure 2. Image of the planet's surface based on VENERA-9 panoramas of the landing site (1975) in modern processing. 


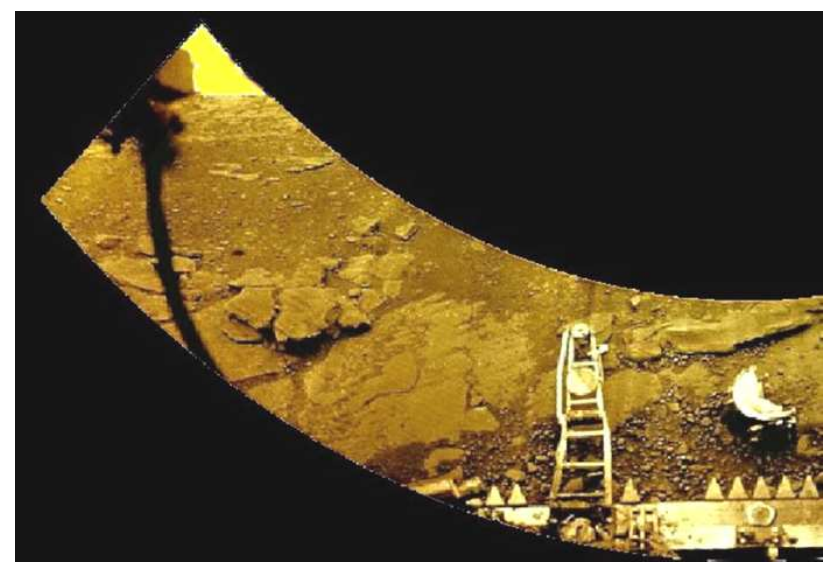

Figure 3. Left potion of VENERA-13 panorama taken by camera 1 after processing with up-to-date techniques. Geometric distortions have been corrected.

The obtained images were black and white, without many details, but rather sharp (Figure 2). More sophisticated experiments were placed onboard the VENERA-11 and -12 spacecraft in 1978. To protect the lens inputs of the TV cameras during the descent, there were lids covering optical inputs. The lids had to be removed by special pyropatrons after landing. However, the experiment failed: as it turned out, some properties of the Venusian environment were not taken into account at the design of the landers, and the lids were not detached. For more than an hour, the cameras sent to Earth images of the inner surface of the lids. The view of the surface at the landing sites remained unknown. Later, it was reported that there were also problems on the VENERA-9 and -10 landers (1975): the lids were detached only on one of the two cameras on each of the landers.

(a)

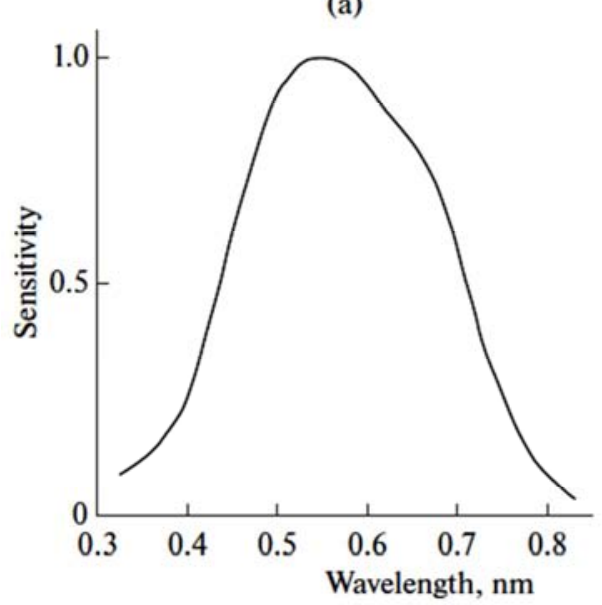

A genuine triumph of researchers was the VENERA-13 and -14 landers (March 1982) [4]. The detailed color panoramic images of the surface of Venus (Figure 3 ) transmitted by the landers were published all over the world. The opticalmechanical TV cameras of the landers [2, 3] were designed by a team of specialists from the Space Systems Institute led by coauthors of this paper, Dr. Selivanov and Dr. Gektin. By the start of the work on TV cameras for the VENERA landers, Selivanov's team had already designed the TV-cameras for the $L U N O K H O D$ rovers and Mars landers.

The TV-cameras themselves were unusual in design. At that time, arrays of semiconductor detectors of images, CCDs (charge-coupled devices), which are currently used in electronic photographic cameras, had not been developed yet. In the 1960s-1970s, the lunar and Martian spacecrafts were equipped with film cameras containing automatic devices for chemical developing, electronic scanning, and transmission of the obtained images by radio link to Earth. However, in the TV cameras of the VENERA landers, where the temperature was continuously increasing, it was impossible to use a photo process. The use of ordinary TV camera tubes, unsuitable for operation under high temperatures, was not only more risky, it required significant volume of onboard memory. So it was decided to use a one-channel electronic device, a FEU-114 photomultiplier and a real-time radio link. The spectral characteristic of the photomultiplier's photocathode corresponds to the multialkali type (Figure 4a); the wavelengths at the 0.2 level of the maximum (at $550 \mathrm{~nm}$ ) are 400 and $760 \mathrm{~nm}$. In front of the photomultiplier's cathode, there was the opaque screen with a diaphragm. The lens formed an image on the screen, and an image element that entered the aperture was transmitted as a pixel signal.

(b)

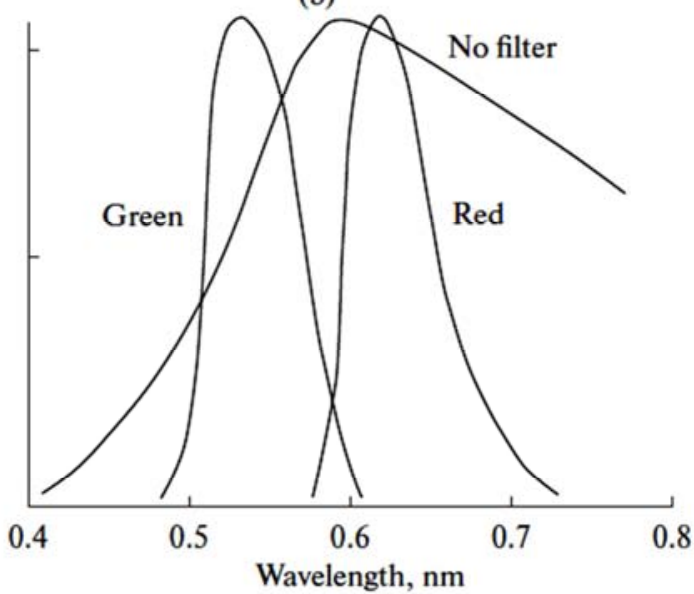

Figure 4. Spectral characteristics of TV-cameras of VENERA-9 and -10 (a) and VENERA-13 and-14 (b) landers.

The aperture size was 11 arc min. Thus, the resolution of the panoramas of Venus cedes to normal human vision by 22 times.

It should be mentioned that a direct return of the scientific data from Venus surface to Earth was a problem. Instead VENERA satellites were used as re-translators. One full image of the VENERA-13 and -14, a "panorama", extending from horizon to horizon, took 13 minutes to be completed and transmitted. For 2 hours of active functioning the TVcameras duplicate images up to eight times. The clarity of a picture element that has a fixed size depends on the distance. The (vertical) line resolution was 211 pixels and 11' (arc $\mathrm{min})$; thus, a pixel size of $0.5 \mathrm{~cm}$ corresponds to the distance $0.005 /(11 / 3438)=1.56 \mathrm{~m}(3438-$ the number of minutes in 
one radian). Unfortunately, upon heating, the equipment's adjustment deteriorated, and the actual resolution became worse.

The area surrounding the landing site was reflected by a mirror mounted in front of the lens, and the mirror swung within an angle of approximately $40^{\circ}$. In the VENERA-13 and -14 and VENERA-9 and -10 cameras, one sweep of the mirror took 0.78 and $3.5 \mathrm{~s}$, respectively. In such a way, one picture line whose elements passed by turns through the diaphragm, was formed. In contrast to a usual TV, lines were vertical; at the end of each sweep, a special mechanism rotated the mirror by an angle corresponding to the aperture size, in the plane perpendicular to the sweep direction and a next line of the picture was formed. In the VENERA-13 and 14 cameras, a whole image contained 1000 lines, while a line itself contained 211 elements of the image (pixels) and 41 elements of the service information. (The image transmitted by the VENERA-13 lander and processed with up-to-date methods is shown in Figure 3.) To protect the camera from thermal radiation, a periscope system was used that placed the camera at the lower end and the scanning mirror at the top. The VENERA-13 and -14 cameras transmitted split-color images obtained with red, green, and blue filters (Figure $4 \mathrm{~b}$ ) (rather than only black and white of the VENERA-9 and -10 cameras). However, since the radiation in the blue spectral range is almost completely absorbed by the atmosphere, the blue panoramas were worthless and were not used. To scan a whole image took $13 \mathrm{~min}$.

Though the guaranteed operation time of the VENERA-13 and -14 cameras was 30 min they worked successfully for $2 \mathrm{~h}$ and transmitted a considerable number of panoramas and their details. The images and service information were coded in a 10-bit system (1024 levels in total, where 512 levels were for the image) and transmitted via the omnidirectional antenna of the lander to the orbiter. Each of the landers was equiped with two cameras mounted on its opposite sides. The resolution in the VENERA-9 and -10 panoramas was almost two times worse than that of the VENERA-13 and -14 cameras. Their cameras produced only black and white pictures. The 180-degree panorama contained 517 vertical lines of 115 pixels each, and it took approximately $30 \mathrm{~min}$ to obtain a complete image. For the VENERA-9 and -10 the angular resolution (corresponding to a single pixel) was $21^{\prime}$ [2]. The input of the optical system was placed $82 \mathrm{~cm}$ above the landing buffer for the VENERA-9 and -10 and $90 \mathrm{~cm}$ at VENERA-13 and -14 probes.

The VENERA-9 and -10 landers worked on the surface of the planet for 50 and $44.5 \mathrm{~min}$, respectively. The VENERA-9 panorama actually covered $174^{\circ}$ and the duration of imaging (with its real-time transmission) was $29.3 \mathrm{~min}$. After that, the right portion of the panorama, within $124^{\circ}$, was taken again. The coverage of the VENERA-10 panorama was $184^{\circ}$; two fragments at the beginning and the end of the image, by $63^{\circ}$ and $17^{\circ}$, were subsequently repeated. The image was coded in a 6-bit system (64 levels), transmitted via the omni directional antenna of the lander to the orbiter (in a 48-h orbit), and relayed in real time to the Earth by its narrow beam antenna.

It should be mentioned that a direct return of the scientific data from Venus surface to Earth was not impossible, ruther it would be useless due to the low signal-to-noise ratio. Instead the VENERA satellites were used as re-translators. One full image of the VENERA-13 and -14 cameras, a "panorama", extending from horizon to horizon, took 13 minutes to be completed and transmitted. For 2 hours of active functioning the TV-cameras duplicate images up to eight times. Unfortunately, upon heating, the equipment's adjustment deteriorated, and the actual resolution became worse. If the image of a specific object is not single then batch processing and stacking can be used to study the details. Methods of treatment of a single low-noise image can increase its clarity substantially.

\section{Chemistry and Physics of the Atmosphere and Surface at Venera Landing Sites}

The special issue of "Kosmicheskiye Issledovaniya" ("Space Research"), V. XXI, No. 2-3, 1983, presented the main results of the VENERA-13 and -14 missions. The coordinates of the VENERA-13 (March 1, 1982) landing site were $7.5^{\circ} \mathrm{S}, 303.5^{\circ} \mathrm{E}$, and its height above the level of nominal radius $6051 \mathrm{~km}$ was $1.9 \mathrm{~km}$ [5]. The temperature was $735 \mathrm{~K}\left(462^{\circ} \mathrm{C}\right)$ and the pressure was $8.87 \mathrm{MPa}$, which corresponds to the atmospheric density $59.5 \mathrm{~kg} / \mathrm{m}^{3}$. The local time was $10 \mathrm{am}$, and the solar zenith distance was at an angle of $37^{\circ}$. Illumination by the diffused sunlight was 3-3.5 kLux.

The lander VENERA-14 (March 5, 1982) sank at the equatorial zone too, at $13^{\circ} \mathrm{S}, 310^{\circ} \mathrm{E}$, and the landing site's height was $1.3 \mathrm{~km}$ above the radius of $6051 \mathrm{~km}$. The measured physical conditions were as follows: temperature $738 \mathrm{~K}$, pressure of $9.47 \mathrm{MPa}$ and atmospheric density approximately $65 \mathrm{~kg} / \mathrm{m}^{3}$. Gas analyzers repeated that the atmosphere is composed almost entirely of $\mathrm{CO}_{2}(96.5 \%)$ and $\mathrm{N}_{2}$ (3.5\%). Local time was also at approximately $10 \mathrm{am}$, with a solar zenith angle of $36^{\circ}$. The scene illumination reached 3.5 kLux [3, 6]. Totally on March 1 and 5, 1982, experiments in television photography instrumented by the landers VENERA-13 and VENERA-14 yielded in 37 panoramas (or their fragments) of the Venus surface at the landing sites.

According to current concepts, the Venusian atmosphere at the altitude range of 0-49 $\mathrm{km}$ does not contain much aerosol; a visibility of the surface is restricted mainly by Rayleigh scattering. Although it is not uniform, high transparency of the atmosphere close to the Venusian surface was observed in all in situ experiments. Inasmuch as the physical conditions for water on the planet are supercritical, Earth-like waterbased precipitates have to be ruled out.

During the years of intense research on the planet by the VENERA and Pioneer Venus probes, some important publications on the thermochemical cycles of interaction between the atmosphere and the surface of Venus appeared. Venus' surface is 5-20 times richer in sulfur as compared 
with the Earth. Because of the huge mass of the atmosphere, its dynamic features, and lack of seasonal effects, local variations of the temperature at the planet surface at a given altitude are negligible and, in fact, represent a natural thermostat.

On top of $\sim 0.965 \mathrm{CO}_{2}$ and $\sim 0.035 \mathrm{~N}_{2}$, the gas environment at the VENERA-13 and VENERA-14 landing sites includes the following minor consituents: $1.5 \times 10^{-4} \mathrm{SO}_{2}, 2 \times 10^{-5} \mathrm{H}_{2} \mathrm{O}$, about $2 \times 10^{-5} \mathrm{O}_{2}$, traces of COS, chlorides $\left(0.4 \times 10^{-6} \mathrm{HCl}\right)$ and fluorides [7-9]. There could be also traces of metals in the gas phase [10].

Following completion of the VENERA 9 and 10 lander missions, Yu. Gektin and A. Panfilov, the TV-experiment coauthors, were among the first researchers who considered models of the thermochemical equilibrium of the gaseous components in the Venusian atmosphere within the range from the surface to the altitude of $30 \mathrm{~km}$ [11]. More than 180 equilibrium chemical reactions between small components of the atmosphere were regarded, including $\mathrm{NO}, \mathrm{NO}_{2}, \mathrm{SO}_{2}, \mathrm{SO}_{3}$, $\mathrm{COS}, \mathrm{H}_{2} \mathrm{~S}, \mathrm{CS}_{2}, \mathrm{NH}_{3}, \mathrm{CN}, \mathrm{C}_{2} \mathrm{~N}_{2}$, etc. As to other Russian publications of the period, V. P. Volkov should be noted [12]. He formulated the chemistry of many natural processes on Venus. The researcher points out that the near-surface part of the troposphere is in a state of a high-temperature chemical equilibrium with the surface rocks, and the redox activity of the surface is determined by the solid phase mineral buffer 'pyrite-anhydrite-magnetite' that is independent from the amount of silicon, aluminum, and iron in local rocks. Volkov suggested some basic scenarios of the Venus hightemperature atmosphere-surface chemical interactions, which include, for example, the following reaction:

$$
3 \mathrm{FeMgSiO}_{4}+\mathrm{CO}_{2}=3 \mathrm{MgSiO}_{3}+\mathrm{Fe}_{3} \mathrm{O}_{4}+\mathrm{CO}
$$

(olivine + carbon dioxide $=$ enstatite + magnetite + carbon monoxide), as well as its various options.

During the same period, a number of other writers also considered possible scenarios of the atmosphere-surface interactions [13, 14]. The latter paper examined some processes of rock weathering: in particular, the reaction between mineral fayalite $\mathrm{Fe}_{2} \mathrm{SiO}_{4}$ and carbonyl sulfide COS, a minor atmospheric component:

$$
\mathrm{Fe}_{2} \mathrm{SiO}_{4}+4 \mathrm{COS}<==>2 \mathrm{FeS}_{2}+\mathrm{SiO}_{2}+\mathrm{Fe}_{3} \mathrm{O}_{4}+2 \mathrm{CO}_{2}+2 \mathrm{CO}
$$

with the reaction shifting to the right at lower temperatures. In other words, pyrite $\mathrm{FeS}_{2}$ proves to be stable in mountains. Of course, it would be naive to expect that all the basic chemical chains could be established at this early stage of research. The chemistry of the atmosphere and the surface of Venus is studied by many researchers e.g., [9, 12-15]. No inferences concerning possible phase transitions of the compounds near the surface, at the temperatures of $730 \mathrm{~K}$, were found in the aforementioned (or subsequent) publications. Chemical composition of the soil at the landing site is close to that of the Earth' tholeitic basalt [7] shown in
Table 1. (The data were not comfirmed).

\begin{tabular}{|c|c|c|}
\hline Oxides & VENERA-14, \% & Tholeitic basalt, \% \\
\hline $\mathrm{SiO}_{2}$ & $48.7 \pm 3.6$ & 50.6 \\
\hline $\mathrm{TiO}_{2}$ & $1.25 \pm 0.41$ & 1.2 \\
\hline $\mathrm{Al}_{2} \mathrm{O}_{3}$ & $17.9 \pm 2.6$ & 16.3 \\
\hline $\mathrm{FeO}$ & $8.8 \pm 1.8$ & 8.8 \\
\hline $\mathrm{MnO}$ & $0.16 \pm 0.08$ & 0.2 \\
\hline $\mathrm{MgO}$ & $8.1 \pm 3.3$ & 8.5 \\
\hline $\mathrm{CaO}$ & $10.3 \pm 10.2$ & 12.0 \\
\hline $\mathrm{Na}_{2} \mathrm{O}$ & $2.4 \pm 0.4$ & 2.4 \\
\hline $\mathrm{K}_{2} \mathrm{O}$ & $0.2 \pm 0.07$ & 0.1 \\
\hline S & $0.35 \pm 0.28$ & $0.07 \pm 0.01$ \\
\hline $\mathrm{Cl}$ & $<0.4$ & 0.01 \\
\hline
\end{tabular}

Table 1. Comparison of the soil composition.

Of importance is a question about the sources of energy for the hypothetical Venusian biosphere (considered in following sections). The interest in the hypothetical autotrophic flora of the planet as a source of the existence of its fauna was noted in $[16,17]$. Although the direct rays of the Sun, as a rule, do not reach the surface of the planet, there is enough light, say, for the Earth-like type photosynthesis there. In the case of the Earth, a diffuse illumination of 0.5-5 kLux is sufficient for photosynthesis even in the depths of the dense forests. The measured illuminance on Venus is of the same order, at the range of 0.4 to $7 \mathrm{kLux}$. Of course, hypothetical photosynthesis at high temperatures and in a non-oxidizing environment should be based on a completely different, unknown biophysical mechanism.

The daytime and nighttime durations on Venus are 58.4 Earth days each. The day illuminance reached $3.5 \mathrm{kLux}[3,4$, $6]$ and even attains 5-7 kLux. Very special is spectral composition of illumination (Figure 6) that is connected directly with a content of this paper.

The atmosphere absorbs blue rays and the sky is of a yellowish color. The solar disk is hardly distinguished through the permanently present high-altitude cloud layer (above $50 \mathrm{~km}$ level). The cloud layer is formed by micronsized droplets of concentrated sulfuric acid. A color of a day illumination is like as in Figures 2 and 3.

The known requirements of the terrestrial type photosynthesis to the spectral features of the absorbing radiation is difficult to reconcile with the measured spectral distribution of solar radiation at the surface of Venus (Figure 5, 6; the vertical scale is logarithmic). The data were obtained in spectral experiments on VENERA-14 lander [6]. The shortwave optical radiation in the range $410-500 \mathrm{~nm}$ is suppressed almost completely by the planet's atmosphere. Illumination in the orange-red region $(600-720 \mathrm{~nm})$ reaches 5 kLux. In the near-infrared illumination on Venus is high also, but drops sharply at a band of water vapor ( 0.93 microns). 


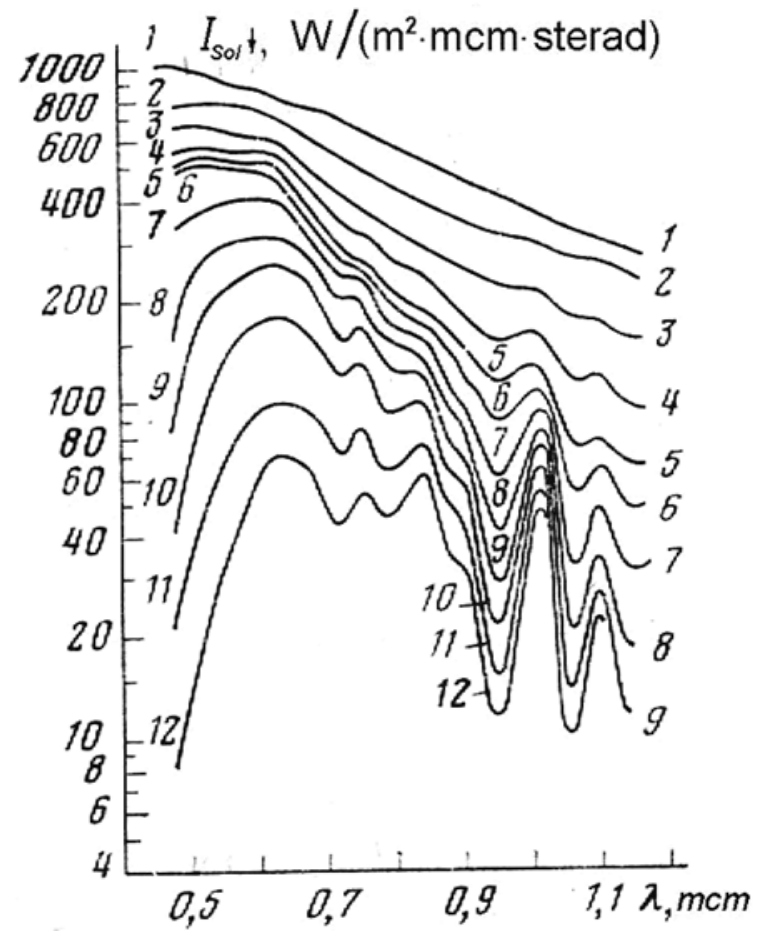

Figure 5. Scattered solar radiation spectra measured by the VENERA-14 spectrometer in the atmosphere and at the surface [6]. 1 - above clouds, 2 at $62 \mathrm{~km}$ altitude, $3-55,4-52,5-49,6-40,7-25,8-16.5,9-10,10-$ $4.5,11-1,12$ - at $0 \mathrm{~km}$.

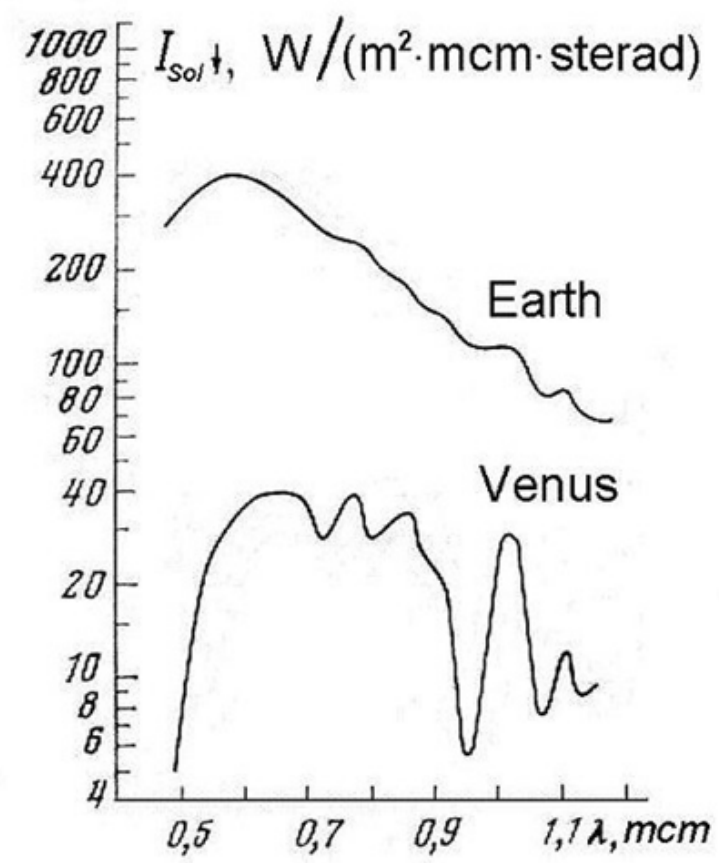

Figure 6. Intensity and spectral distribution of the solar radiation at surfaces of Earth (clean sky) and Venus.

\section{Hypothetical Plants on Venus}

\subsection{The First Hypothetical Plant Found on Venus}

After the panoramas were processed with the software available in 1982-1984, the results were published in [16-18] and other journals. Though 35 and 42 years have elapsed since the time of the surface panoramas were obtained, the experiment has not been repeated by the Russian or any other space agency of the world, as mentioned above.
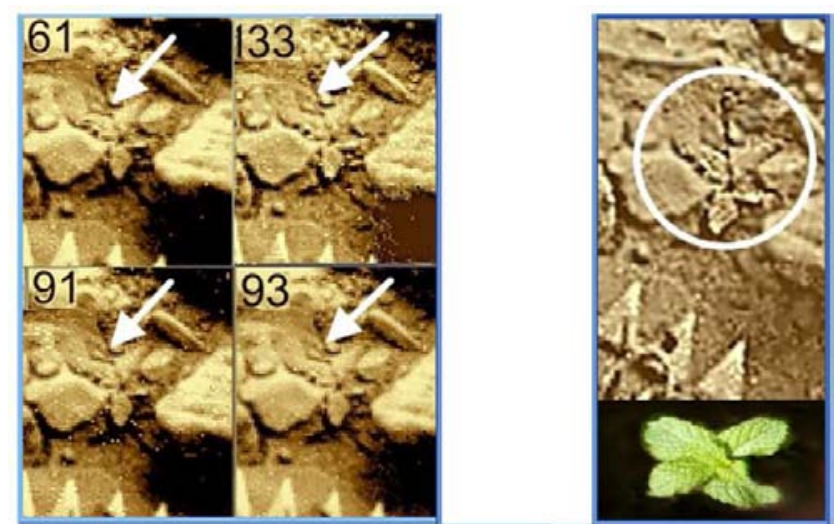

Figure 7. Left: 4 repeated fragments of the surface image at the landing site of VENERA-14. "Scratches" are shown by arrows. Right: Stacked image of the first found "stem" that is a thin vertically arranged trunk that has a height of approximately $40 \mathrm{~cm}$ and a thickening ("burgeon") on the top. At its base there are features that resemble leaves of an Earth quatrefoil plant. The "stem" is located at a distance of approximately $40 \mathrm{~cm}$ from the landing buffer of the VENERA-14 lander and is seen from above. Below leaves of an Earth' quatrefoils are shown, for a comparison of their size and shapes.

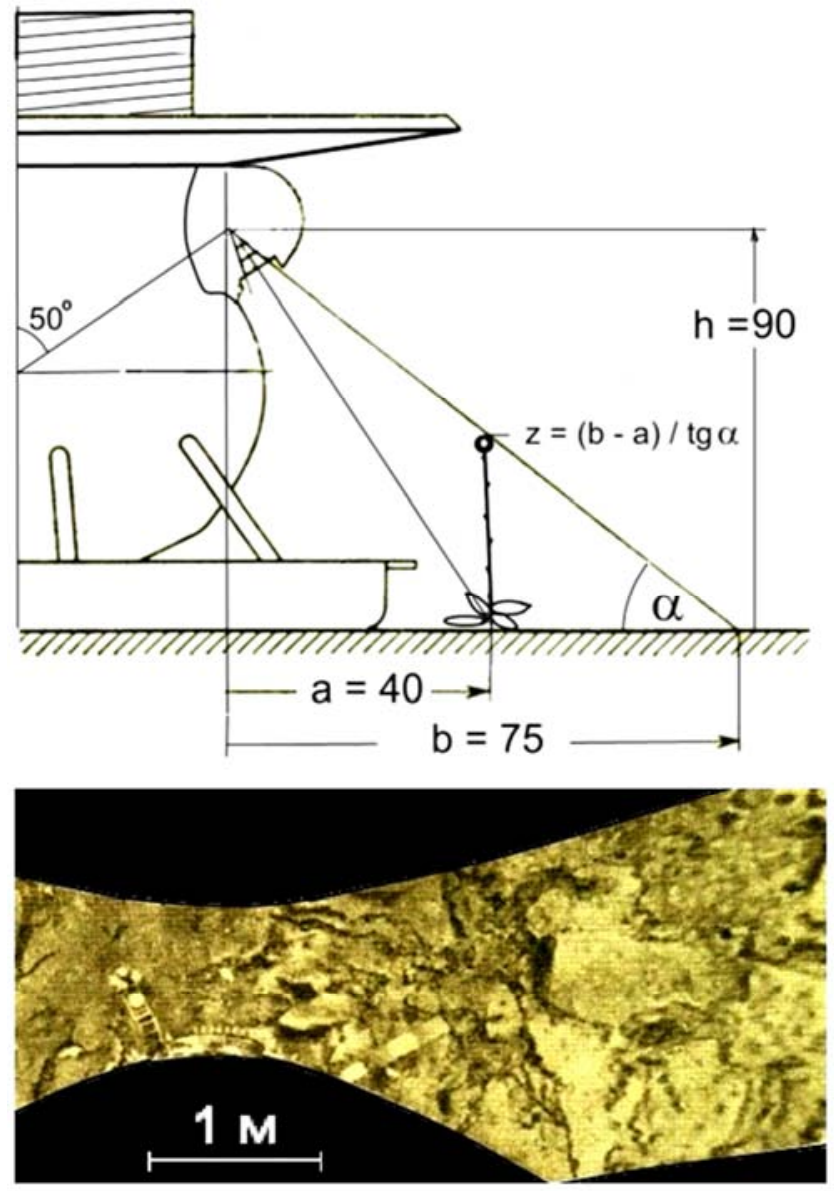

Figure 8. Geometric scheme used to determine sizes of plant (sizes are in centimeters) and fragment of VENERA-14 photomap. 
Meanwhile, advances in software and image processing have allowed the quality of the panoramas to be substantially improved. The purpose of the first analysis [19] and other works was to find any differences in the sequential panoramas (appearance or disappearance of details or changes in pattern) and to understand what might have caused them (e.g., wind).

One more sign of the objects of interest is the peculiarities in their morphology that makes their shape differ from common geologic formations. Not all of the images are identical in contrast and sharpness, which requires special processing for their comparison. In the original images, the "stem" attracted no attention for a longtime, as in unprocessed panoramas it is hardly distinguishable; in the processed images, it resembles a weakly noticeable defect, or a scratch, though repeatedly occurring in all of the images at the same place. In Figure 7, the same location of the "defect" is indicated by arrows.

Figure 7 (the left part) shows how barely noticeable objects repeats in properly processed panoramas. After the images had been additionally processed, instead of a scratch, one could observe an object (Figure 7 , in a circle) that exhibits the evident similarity to terrestrial plants. The first stem object was detected due to its being close to the entrance of the TV-camera, and others were detected by similarities in their shapes and positions to the first stem. What was found are vertically standing knotty black stems. Each panorama was returned for $13 \mathrm{~min}$; for many fragments there are up to 8 images obtained independently during 2 hours. When processed, the "stems" are vertically arranged thin knotty trunks, which are $0.3-2 \mathrm{~cm}$ thick and 0.2-0.5 m (and may be more) tall. At the base of the 'stems', there are features that resemble leaves in a quatrefoil. Each of the "leaves" has a size of approximately $5-10 \mathrm{~cm}$, and possibly, they have a radial structure (Figure 7 , right part). On panoramas, stems look black. The first object that was detected (Figure 7), has a large bulge at the top end, a "burgeon", with a lighter center.

To find the height $z$ of the stem, one should use geometric relations (Figure 8) and a photoplan (because, on the original panoramas, the distances are significantly distorted). The input window of the TV camera is located at a height of $h=$ $90 \mathrm{~cm}$, the distance $a$ from the projection point of the TVcamera lens onto the surface, to the base of the stem is approximately $40 \mathrm{~cm}$, and the top of the stem is projected onto the surface details, roughly at the distance of $b=75 \mathrm{~cm}$. If the stem is placed vertically, from the right triangle the angle $\alpha$ at its apex is found from $\operatorname{tg} \alpha=b / h$, and the stem height is $z=(b-a) / \operatorname{tg} \alpha=42 \mathrm{~cm}$. An error is possible as the ground surface is uneven.

\subsection{Stems with Flowers}

To search for other stems, an additional processing of the VENERA-13 and -14 panoramas has been made to improve the clarity of details. At the VENERA-13 landing site, only one or two "stems" were found, for which the base of the "stems", similar to in Figure 7, were in a crack between the stones. This circumstance can be important because the soil here is mainly fragmented, but the stems there were not found. This interesting object is shown in Figure 9, which presents four consecutive images of a stem that was found in the VENERA-13 panoramas. The "stem" in Figure 9 is lower than in Figure 7; it is more distant, and the stem itself is not easy to notice, although there are eight distinct images (duplicates), which allows for batch processing. The attention is drawn to the top of the stem, which appears in each part of Figure 9 as a triad of bright dots that are visible on all of the original high-contrast images. The position of the triad is not identical in successive frames. It varies slightly with respect to the adjacent light-colored stone on top of it. As was shown by the animation, changes arise from the swinging of the triad by the wind. The wind speed is low, about $0.4 \mathrm{~m} / \mathrm{s}$, but the gas density is high.
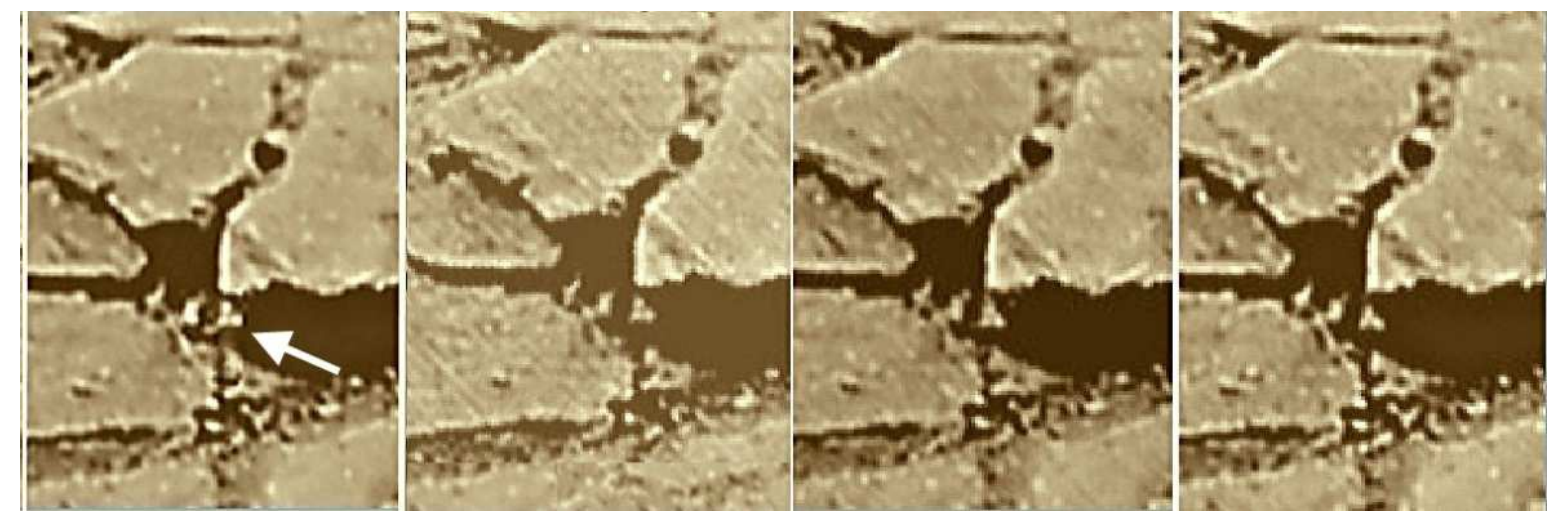

Figure 9. 4 consecutive images of the landing site of VENERA-13 obtained within 1 hour. Arrow in the foreground points "a stem" with a "flower".

A clearer picture of the stem is highlighted by the circle in Figure 10. The top of the "stem" is more complex than the triad (or bud in Figure 7). The object is visible from above, and its height, which is found by its position on the photoplan, is only approximately $20 \mathrm{~cm}$ above the base in the crack between the stones.

At its base, there is a group of four bright details, similar to the "quatrefoil" leaves shown in Figure 7 that appears to be associated with the stem, too. It has been suggested that the complex structure of the top of the stem is an opened 
burgeon. When processing the image decreasing a contrast, this assumption was confirmed and allowed us to see the whole "flower" of a regular shape (Figure 10, right side), with a white spot (pestle?) in the center and the surrounding petals. Fine details appear.

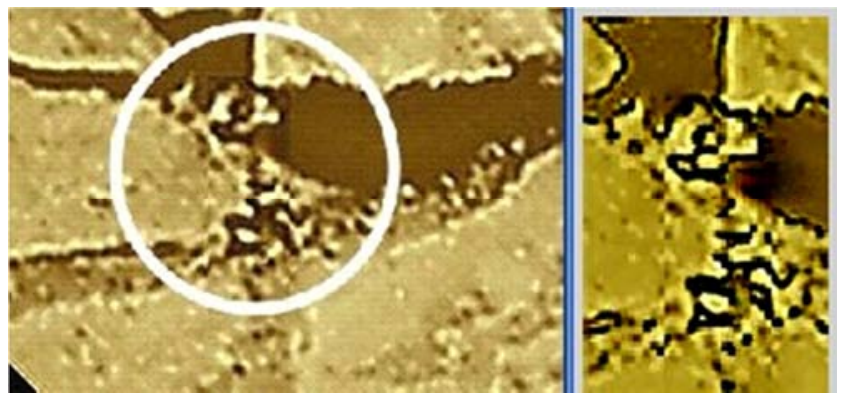

Figure 10. Flower - the same object as in Figure 9, with gradually lowered contrast, from left to right. The detailed image of the "flower" shows its light central part and leaves at the base. The diameter of the flower and the "quatrefoil" at the base is $5-8 \mathrm{~cm}$.

The flower is composed of six to eight light petals. Its right-hand bright part forms the triad that is repeated on all of the duplicates in Figure 10, as part of an open flower. The flower size $(5-8 \mathrm{~cm})$ is approximately the same as a "quatrefoil" at the base of the stem. The VENERA-13 panorama has been organized in such a way that Figures 9-10 represent only a fragment of the black-and-white image; thus, one can talk about only the bright colors of the petals, and their color in Figure 10 is unknown.

Another interesting but unobtrusive very small bright quatrefoil was detected at the center of the VENERA-14 panoramas (Figure 11, see frames 1 and 2) in a depression that is near to the landing buffer, opposite side to the Figure 7.

In contrast to Figure 7, its "leaves" are very bright (young?), only slightly darker than the white tooth of the lander's buffer. One of the quatrefoil elements is in the shadow of a stone. The dimensions of the "leaves" are not more than $2 \mathrm{~cm}$. Despite its smaller size, the object similarity with Figure 7 is obvious. The "stem" itself is difficult to notice on the source panoramas (frame 1); it was isolated by using a gamma-correction (shown in Figure 11, column 3 as consecutive original pictures). The final version is seen in frame 4.

The height of the plant observed from above is approximately $10 \mathrm{~cm}$. There is a "flower" seen on its top, also. When the image is processed, the "stem" gets viewed as in Figure 11, frame 4. The dimensions of the "flower" are approximately $2 \mathrm{~cm}$, too. To the right of it, another "flower" is visible, the stem of which apparently is placed behind the stone.

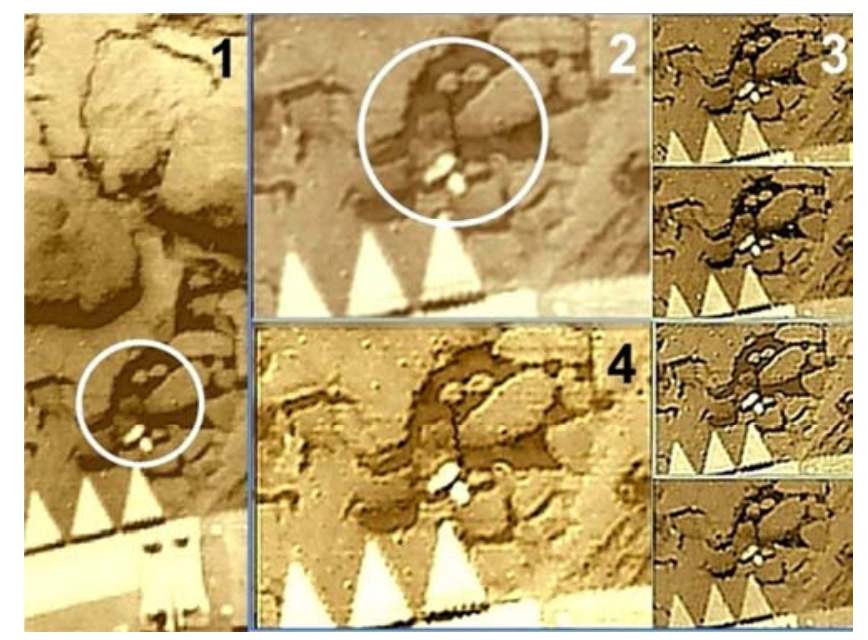

Figure 11. Stem $(1,2)$ with a bright "quatrefoil" located directly at the landing buffer of VENERA-14; its recurring images are shown on four consecutive panoramas (column 3). The processed image is shown in frame (4). To the right of the "flower"; there is another "flower" visible, the stem of which is situated behind the stone.

In Figure 11, small "stem" and its "flower" are seen against the background of contrasting details and cracks in the stone slab recess. The stem rises from the recess. The objects are relatively close to the camera (less than $1 \mathrm{~m}$ ), and compared with Figure 7, the resolution is the same, but the "flower" is small.

\subsection{On the Possible Role of Burgeons and Flowers}

The landing site around the landers VENERA-13 and VENERA-14 showed rare vertically oriented objects that were similar to the stems of terrestrial plants. Another similar stem was found in VENERA-9 image. Probably, the 'stems' are widespread on the planet, because they are met at landing distances between the three different VENERA missions sites that were 900 and $4500 \mathrm{~km}$ apart. The both VENERA-14 cameras registered stems at opposite sides of the lander. The VENERA-9 and VENERA-13 cameras registered stem at one side only. The stems are important objects of a hypothetical Venusian flora discussed in [16-19 et al.]. If the tops of the stems really are burgeons and flowers, one should reflect their role. The flowers of terrestrial plants are intended for their pollination and reproduction. Pollination is conducted either by insects or by the wind. Wind-pollinated plants do not require blooms in principle, for example, the case of the poplar "fluff." Flowers attract insects. Do the tops of the stems in Figure 7, 10 and 11, at least indirectly, hint on the likely participants in the process of pollination? 


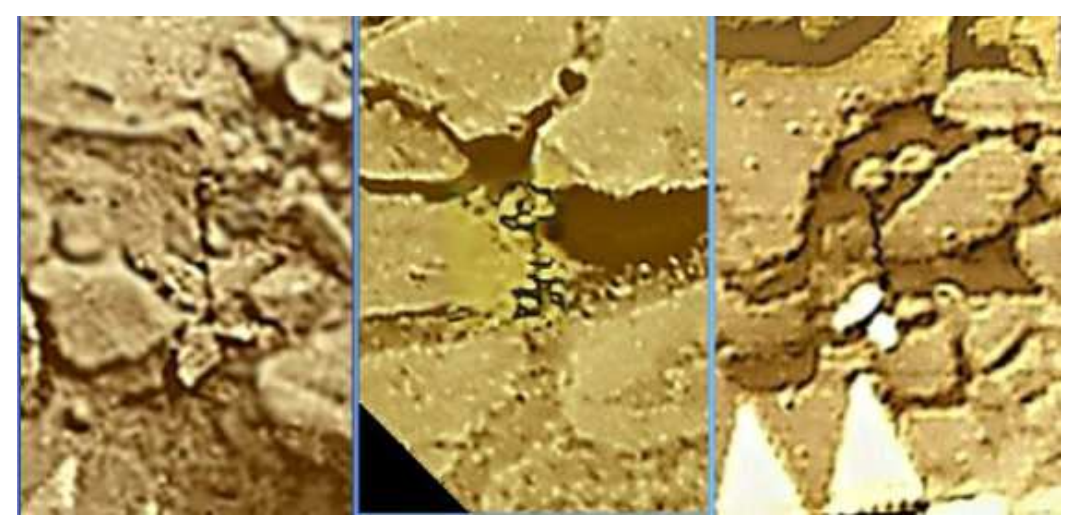

Figure 12. 3 stems with "buds" and "flowers" found in different landing sites.

Terramorphism of hypothetical objects of the flora and fauna of Venus was observed repeatedly in many entities [1619 et al.], for example, a terramorphic is object "mushroom", not presented here. Flowers with their petals in Figure 12 are new objects that are surprising to find, and it is surprising to find the occurrence of the same forms of living objects on different planet that have radically different physical settings. However, what are the laws of nature that determine the recurrence of terramorphism hidden in such markedly different environments?

The high-density hypothetical habitability of Venus [1620] suggests that its surface is more similar to the bottom of a sea shoal on Earth than on the Earth's surface. If the autotrophic flora is a possible source of energy that could be used by the hypothetical fauna of the planet, then one may think that the detection of "stems" would be corroborated. However, this autotrophic nature itself does not solve the problem of feeding to the fauna by these very rare plants. Smaller vegetation, such as grass or moss, is not resolvable in the VENERA pictures. Although the direct rays of the Sun, as a rule, do not reach the surface of the planet, there is enough light for photosynthesis of the Earth-like type there. In the case of the Earth, a diffuse illumination of 0.5-5 kLux is sufficient for photosynthesis even in the depths of the dense forests. The measured illuminance on Venus is of the same order, at the range of 0.4 to $7 \mathrm{kLux}$. Of course, photosynthesis at high temperatures and in a non-oxidizing environment should be based on a completely different, unknown biophysical mechanism.

\section{Hypothetical Fauna}

The identification of various objects on the surface of Venus as examples of Venusian flora and fauna is hypothetical. The (current) names proposed for them, such as Scorpion, BearCub, and others, remain conditional. For the sake of convenience, we have proposed for all objects of hypothetical fauna the general term "hesperas" (originating from the ancient Greek name of Venus), and the term "stems" for plants (objects of Venusian flora), respectively.

We cannot state that all the hesperas and stems available on panoramas of the Venusian surface have already been found. But all large-size and intermediate-size objects, with minor exceptions, have already been presented. Few of a dozen of Venusian fauna objects are presented below.

\subsection{Amisada}

One of the most interesting recognized hypothetic objects related to hesperas is the "amisada" (nickname) that turned out close to the input window of the VENERA-14 TV camera. The conditional term "amisada" originates from the shortened name Ammizaduqa of the Babylonian king (sixteenth century BC). At that time, ancient astronomers started to register on clay tables the time of morning and evening elongations of Venus.

The shape of the amisada resembles a reptile climbing up a stone. The amisada were located in the immediate vicinity of the TV-camera lens; therefore, we managed to resolve many of its fine details.

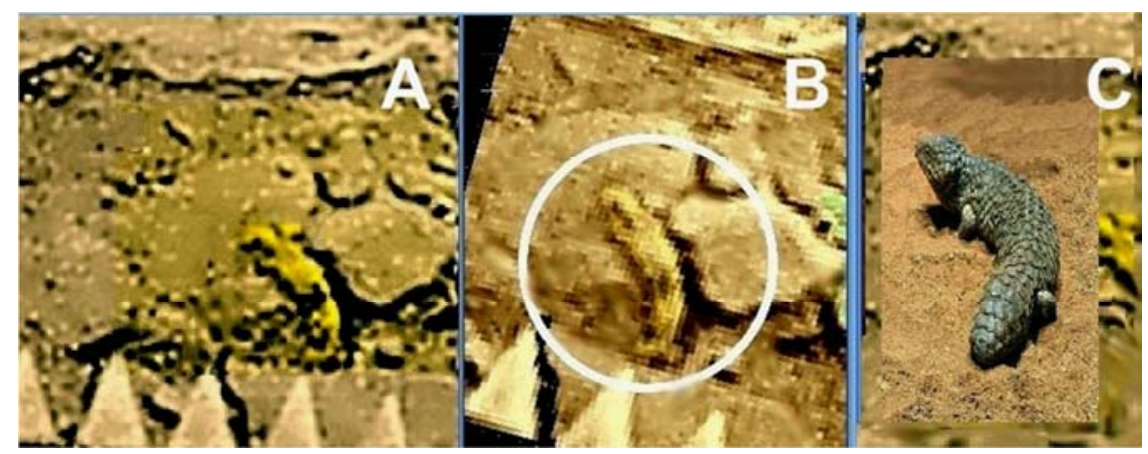

Figure 13. (A) Amisada climbing up a stone, (B) stacking of 6 original fragments of VENERA-14 panoramas, (C) sluggish Australian Shingleback lizard whose size and shape resemble the amisada. 
This amisada is interesting in connection with the fact that by virtue of the low-noise TV images of VENERA-14, it is possible to see and to recognize fine and slow displacements of the amisada's forward part, supposing that its left upper end in Figure 14 is its forward part. In Figure 13A, a single image of the amisada is presented, which corresponds to about the 30th minute of activity of the TV-camera of the VENERA-14 lander. In Figure 13B, the averaged shape (for 6 original images) of the amisada is shown. Further, in Figure 13C, the sluggish Australian Shingleback lizard is also presented, which according to its size $(10-15 \mathrm{~cm})$ and tardieness resembles the amisada. Images of the amisada were additionally processed, which made it possible to demonstrate the motion of its body as a sequence of six sequential positions.

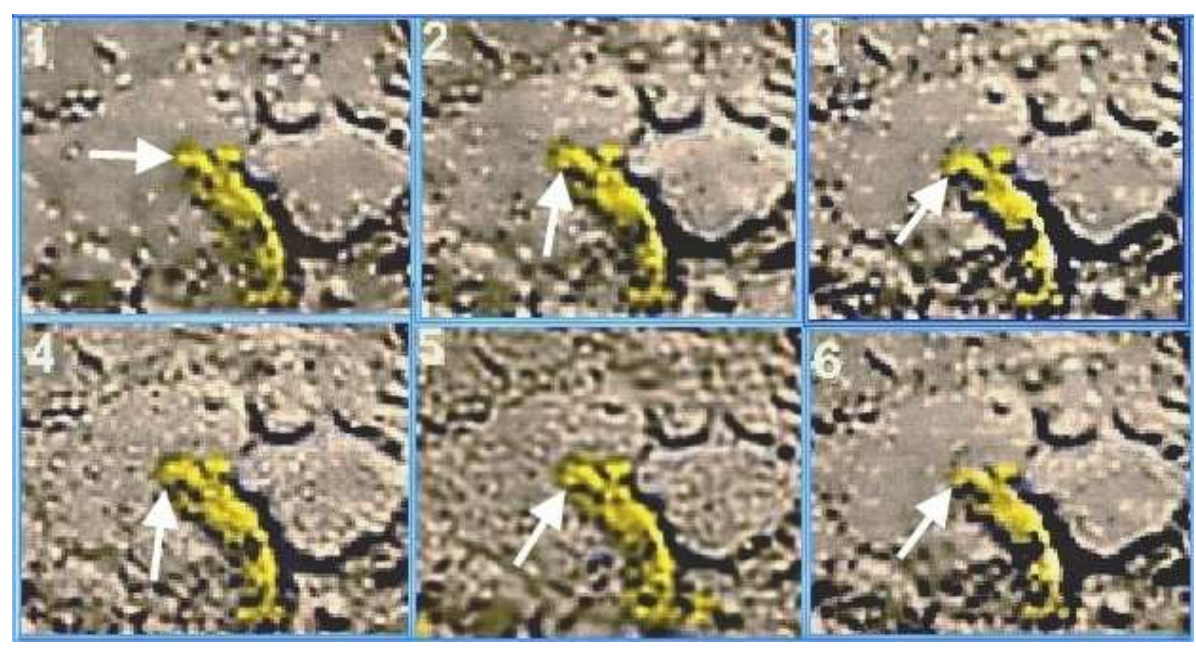

Figure 14. Nonaveraged processed fragments of original panoramas. Positions of arrows indicate the sequentially varied directions of the forward part of the amisada, whose size and motion resemble a human finger. Arrows indicate how the forward part changes its direction. (All images originally are black and white).

Position variation of the amisada is shown in Figure 14 as changes in the direction of arrows repeating positions of its forward part. Here, fragments of the six available panoramas are given. The images are presented in chronological order, with the intervals between them being, on average, of about $13 \mathrm{~min}$. In frame 1 of Figure 14, the upper part fragment of the amisada is directed to the left (the $9 \mathrm{~h}$ ), whereas the shadow under it is almost not visible. We should recall that for scattered natural illumination on the Venusian surface, shadows appear only in the case of low positions of an object with respect to the surface. In this case, the altitude of the object above the surface should be comparable to its size. Most likely, the absence of a shadow indicates that the object's part is lifted above the surface. In frame 2, the amisada's forward fragment is displaced along the $7 \mathrm{~h}$ direction, and no shadow is also present. Finally, in frame 3, the edge fragment corresponds to, approximately, the $8 \mathrm{~h}$ direction with a dense shadow under the object. Further variations are given by the frame sequence 4 to 6 . The size and displacements of the fragment are close to those of a human finger and its motions. In this case, the displacement, in itself, attains 1 to $2 \mathrm{~cm}$ with the speed of observed displacement lower than $1 \mathrm{~mm} \mathrm{~s}^{-1}$. In addition to the amisada, there are two similar objects seen nearby.

\subsection{Bear-Cub}

Terramorphism intrinsic to certain types of possible Venusian fauna, apparently, relates to the deepest puzzles of searching for life in the Universe. If objects existing under quite different physical settings exhibit, nevertheless, significant similarity of their shapes, then this phenomenon should be based on certain general natural regularities not yet understood. In this connection, the object called BearCub (Figure 15) is of interest. This object was found in 2012 upon additionally processing the VENERA-9 panorama (obtained back in 1975).

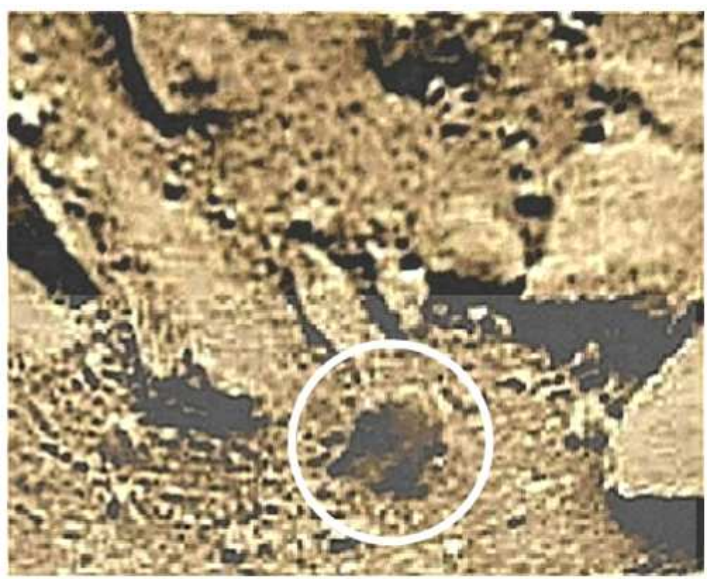

Figure 15. Panorama fragment obtained as a result of the VENERA-9 mission upon additional data processing. In the forefront, there is a small object nicknamed a BearCub. By its soft contours, the object is distinguished from the sharp stone edges. To the left beyond the object, over the ground, there are propagate long furrows that terminate under the BearCub. Apparently, these tracks indicate the preceding motion of the object.

BearCub resides in the forefront of Figure 15 in the lower part of the panorama. In contrast to the sharp contours of the 
surrounding stones, the surface of the object is rather soft and even may be called fluffy. The object is located close to the TV camera at a distance of $0.9 \mathrm{~m}$ from its optical input window and is seen almost from above at an angle of $60^{\circ}$ to the horizon. BearCub seems to rest on its extremities with a certain clearance between them. In this case, as is shown in Figure 15, these extremities resemble animal paws. BearCub is similar in sizes to an animal like a small dog. According to the positions of shadows, which are the same for BearCub and the surrounding stones, one may assume that this object is positioned vertically, with its height and length (width) being 32 to $34 \mathrm{~cm}$ and about $16 \mathrm{~cm}$, respectively. Important details can also be distinguished in Figure 15 to the left of BearCub. Here, four or five long furrows 65 to $70 \mathrm{~cm}$ in length are seen. They start near the left plane stone and envelope a large clod located in the middle part of the figure. The furrows envelop a small object, approximately $5 \mathrm{~cm}$ in size. Further, they extend beyond BearCub and terminate immediately near its extremities. In front of BearCub or, to be more precise, to the right with respect to it, there are no such tracks. Apparently, BearCub is the object that has formed the furrows as a result of its motion. Thus, one is able to estimate extremely important characteristics of the motion, namely, its velocity and, possibly, even the maximum displacement velocity.

The velocity can be determined under the assumption that the object attempting to escape from danger started to move at the instant of close landing of the lander. (The lower part of Figure 15 immediately adjoins the landing buffer of the probe.) The total time of sweeping the panorama is $30 \mathrm{~min}$, whereas the time elapsed between the start of sweeping and the scanner attaining the position of BearCub was about 16 min. Under these extreme conditions, the object leaving 65cm tracks could develop a maximum velocity of $64 / 16=4$ $\mathrm{cm} \mathrm{min}^{-1}$, or less than $1 \mathrm{~mm} \mathrm{~s}^{-1}$. It has been noted at other objects [20] that the displacement velocity intrinsic to Venusian fauna seems to be very low from the terrestrial standpoint.

Is this conclusion sufficiently substantiated? It is not excluded that the velocity of the object was significantly higher. The object could have moved much more rapidly and then have stopped at the point seen in Figure 15. One might substantiate this assumption in the following manner. In the course of the VENERA-9 mission, two panoramas were obtained, the second one being incomplete. It covered only $124^{\circ}$, its quality was much worse than that of the first panorama, and it hardly could be subjected to processing. There, BearCub is not seen so clearly. However, comparison with the first panorama allows making the following conclusion. Within the time of, approximately, $30 \mathrm{~min}$ that elapsed after the first scanning, the location of the object has changed negligibly, and, in any case, has not exceeded several centimeters. Thus, it is quite possible that, under the critical situation, the object initially moved rapidly, and then stopped.

Is it possible to find competing explanations for appearance of the furrows, e.g., the displacement of the object under the action of wind? In [21], the wind velocity at the landing point of VENERA-13 was measured, which reached the value $V=0.40-0.45 \mathrm{~m} \mathrm{~s}^{-1}$. Measurements performed with other modules of the VENERA series have given the same results. The equivalent velocity of the Earth's wind is $8 \mathrm{~m} \mathrm{~s}^{-1}$. The possibility for BearCub to be displaced under the action of wind is determined by the wind head $F=$ $\rho S V^{2} / 2$. For gas density $\rho=64 \mathrm{~kg} \mathrm{~m}^{-3}$ and the lateral surface area of BearCub $S=0.05 \mathrm{~m}^{2}$, the wind pressure force attains $F=0.26 \mathrm{~N}$. This force is insuffiient to shift the object.

The shape of BearCub, which is unusual compared to other objects found, as well as its traces and position resembling Earth's animals in the mobile state, determine its high place among other candidates for Venusian fauna. One may say that alongside with other events, the VENERA-9 panoramas represent a certain demonstration area for these candidates as in the middle part of the panorama one can also see another interesting objects.

\section{3. “An Extraneous Object on the Surface of Stratified Rocks"}

In the special issue of the journal Kosmicheskiye Issledovaniya published in 1983 immediately upon completion of the VENERA-13 and VENERA-14 data analysis, paper [22] by Florenskii et al. was published. This article was devoted to the geological analysis of the landing region. The authors noted clearly pronounced fine subhorizontal layering with the formation of large steplike ledges 1-2 to 5-10 cm high. The layers differed from each other in their reflection ability. Further, the attention of the authors was attracted to an unusual surface segment sufficiently close to the TV-camera lens. The authors wrote: "... in addition to distinctly observed rocks, in certain parts of panoramas, it was possible to see rock segments, where stratification had not been manifested. For example, on the B14-1 panorama, to the left of the termination of the colortest table, a relatively dark spotted (cellular) object was seen. This object resided in a shallow depression of the relief and exhibited a surprising orientation of spots. Apparently, here, from disordered layers of the stratified packet, a structure composed of other-type rock stands out. This rock can both underlie and compose a certain extraneous object contained in this packet. On the left edge of the B14-2 panorama, there are spotted formations, perhaps related to surface manifestations of analogous spotted rocks" (see [22], p. 345). The authors of the paper analyzed other spotted formations on the B14-2 panorama. However, the "spotted cellular extraneous object with a surprising orientation of spots", apparently, belongs to the most surprising finding Florenskii et al. It is a pity that they have not gone further in investigating this small extraneous object.

\section{4. “An Extraneous Cellular Object”: A Snake}

The panorama fragments discussed below exhibit a limited resolution, namely, only 11' per pixel. The "extraneous cellular object" (in accordance with terminology of [22]) 
resides in the right part of Figure 16, to the right of the colortest panel. It is worth noting that both the panel width and the height of each color field are $10 \mathrm{~cm}$. The depression with the extraneous cellular object is located, approximately, at a distance of $25 \mathrm{~cm}$ from the right end of the panel. In Figure 16 the brightness and the contrast of the image are equalized compared to the original image.

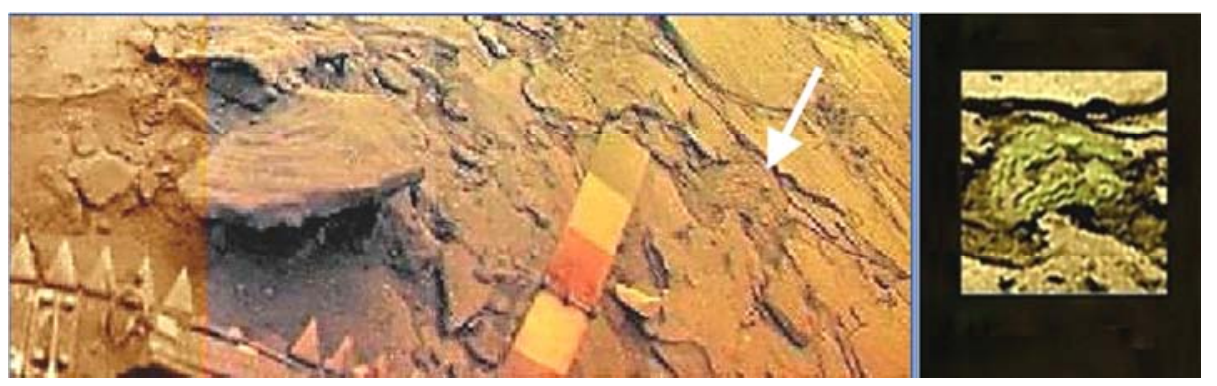

Figure 16. Fragment of the VENERA-14 panorama. The color-test panel is in the central part of the figure. In its right part, coiled snake-shaped object is presented. Its greenish color is exaggerated.

Extraneous object is indicated by the white arrow. The signal-to-noise ratio for color-divided red and no-filter panoramas seems quite satisfactory, which allows us to use individual images in the original form. However, in the case of green images, the ratio was insufficient to have fine distinct details, and, here, we used these fragments only to compare and synthesize the color image. Usually, correct stacking of repeated original images makes it possible to improve the resolution of fine details. Nevertheless, in attempts stacking the images of the "extraneous cellular object", we encountered an unexpected fact. It turned out that, in a number of cases depending on the chosen matching point, certain other parts have turned out to be slightly shifted. The reason for this phenomenon consists in a small displacement of the object parts. At the same time, there are not less than four black-andwhite images, quite suitable for the analysis even in their original form. In addition, there were not less than three of the same color-divided images. Thus, we have managed to form a sequence of isolated fragments as conventional frames of instantaneous photographs for an animation that indicates the object's movements.

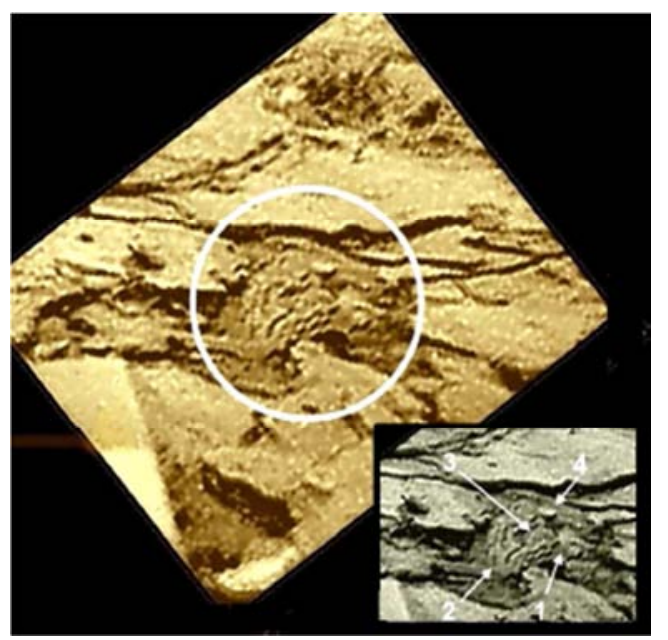

Figure 17. Snake image composed of stacking VENERA-14 frames. For obviousness, the image is turned by an angle of $38^{\circ}$ with respect to the left Figure 16. Below in the insert, the possible snake structure is shown: (1) head with an eye; (2) snake convolved body; (3) crest; (4) tail with an outstanding light detail.
Figure 17 shows an accurately processed image. The surface of the object is covered by regularly located spotted cells. The object is positioned in a small $(5$ to $10 \mathrm{~cm})$ depression and actually resembles a convolved snake (marked in Figure 17 by a white circle). In this case, the object exhibits certain terramorphic features intrinsic to Earth reptiles, e.g., the cellular structure of its surface, in itself, or of its color. The names proposed for other objects found on the Venusian surface, of course, remain conventional. Nevertheless, the name "snake" is the most convenient just from the standpoint of the terramorphic features of the object under discussion. On green images, the snake body is slightly lighter than on black-and-white ones. In other words, the snake has a slightly greenish nuance. The cellular surface of the snake body with its regular spots is strongly distinguished against the background of the surrounding stone plates so that it is barely possible to relate the snake to geological objects. The body is large that makes possible studing its fine details.

In order to estimate the snake size, we make use of the color-test panel shown in Figure 1, 2. The height of each color field is $10 \mathrm{~cm}$, but at the distance of the snake position, the size scale increases by $30 \%$. The total rectified length of the snake attains 40 to $50 \mathrm{~cm}$. In addition to the snake, other unusual objects can be seen in Figure 17. In the $9 \mathrm{~h}$ direction from the snake on a flat light plate, there is a dark formation conventionally called "a dove" whose size attains 5 to $6 \mathrm{~cm}$. The small size of the dove makes it impossible to distinguish its other details besides the shadow under the body and certain fine outstanding parts on the left.

By virtue of the $50^{\circ}$ - tilt of the scanning camera axis, the surface shown in Figure 16 seems inclined. Turning the fragment improves the obviousness of the image, so that the comparison of the size becomes more convenient. Geometric distortions of the images in Figure 16 right part and 17 were not eliminated, but the angle enveloping the figure $\left(34^{\circ}\right)$ is not large; therefore, the distortions are insignificant. Assumptions concerning the snake structure can be made on the basis of Figures 16 and 17. In the insert to Figure 17, a possible interpretation is presented for the snake-body constitution. We can isolate basic parts and elements of snake 
images. The object resides in a small depression; therefore, the entire adjacent surface is covered by the shadow. The basic part of Figure 17 having initial contrast ratios is obtained by stacking the most distinct original images. In the lower part of the figure, the snake structure is presented. We call the extreme right-end part of the snake the "head" (arrow 1). As may be assumed on the basis of the analysis of the fragment shown in Figure 17 and other stacked images, the head part of the object is 6 to $8 \mathrm{~cm}$ in size and resembles the head of numerous terrestrial birds, reptiles, and certain sea animals. Arrow 2 indicates the bend of the convolved body that changes its form in sequential images. Above the neck, there is a crest (3) decorating snake's back. From the bend, the cellular surface extends till the tail (arrow 4). The position of the snake's body changes for few centimeters, from one panorama to another.

The head ends with a sharpened part. By its round "eye", the head is turned to the TV camera. (Here, we call eye the light circular spot also distinguishable in the right part of Figure 16.) The disposition of the light spot leaves nodoubt as to its functions, and we consider as the most likely assumption that here we are dealing with the organ of vision. The arc located to the right and above the eye is transformed to the sharpness resembling a beak.

To the left of the eye, there is a slightly elongated short dark spot that changes its form in sequential images. By analogy with entities on Earth, we may assume that this is the hearing organ (probable acoustic phenomena on Venus were noted in [21]). We may assume that the same organ located symmetrically is also seen. On the left, the lower part of the neck is bounded to properly disposed cells. (Earlier, this fact was indicated by the authors of [22] who wrote that the cells covered the entire forward part of the object). At bend points of the convolved body, a deep fold is observed. This fold has changed its shape in each sequential image.

In the upper part of the neck, there is a detail resembling a crest (3), similar to crests of a rooster. The crest consists of seporated elements and is limited by an arc, about $4 \mathrm{~cm}$ in length. It is the crest that noticeably changes its position relative to the small dark surface segment above the crest. A slight dispersion of images occurs if they are stacked in accordance with certain crest details, or vice versa, the crest is dispersed for other matching variants.

To the left of arrow 3 end, a dark surface of the ground is seen. On sequential images, the crest gradually deviates and screens the dark spot, i.e., the shadowed surface. Its position is different in the right part of Figure 16 and Figure 17. The object head and outstanding parts of the snake body also slightly shift in animated sequential frames.

Apparently, the snake body ends with a long and broad tail that starts from arrow 4 . The position of the snake gradually changes from frame to frame shifting the tail by not less than $10 \mathrm{~cm}$.

The images were obtained for the $1.5 \mathrm{~h}$ lander action on the Venusian surface. As we may conclude, the snake demonstrares small displacements of the body and changes in their positions with respect to other parts of the object or to stone plates on the Venusian surface, which exceeds displacements had been found for other objects. For example, the displacement of the crest in sequential frames for $30 \mathrm{~min}$ attained 3 to $4 \mathrm{~cm}$. Nevertheless, as a whole, the displacement of snake parts is hardly distinguished without attention or application of specific methods.

As was already noted, the image angular resolutionis limited by an angle of 11'. Therefore, it is difficult to recognize fine details on fragments of Figures 16 and 17, which were processed by the unsharp-mask method.

The retarded displacements of the snake testify again to the rather slow type of physical activity of hypothetical Venusian fauna. Apparently, this is associated with energy limitations intrinsic to this fauna as was supposed in [16-20]. At the same time, another probable reason can exist. It maybe associated with the properties of the environment that determines the composition of objects characteristic for Venusian fauna. The presence of liquid water on the Venusian surface is entirely excluded. Hence, entities populating the Venus, if they do exist, should use another medium in their organisms.

\section{Discussion and Conclusion}

The terramorphic features of flora and fauna on other planet, if confirmed, would indicate general laws of living nature, which are not yet known in science. These laws are repeated under the radically different physical conditions including those on the Venus.

Slow displacements observed for the amisada, snake and other objects, confirm once more dilatory character of the physical activity intrinsic to Venusian fauna compared to fauna of Earth. This fact can be naturally associated with energy limitations characteristic of the Venusian fauna evolvedin oxygenless atmosphere. (Let's recall that the Earth's atmosphere also remained oxygenless for more than three billion years). Nevertheless, another likely explanation is also possible. The invariable component of Earth creatures is water, which does not exist on Venus in liquid form. Therefore, in organisms of the possible Venusian fauna, water should be replaced by another medium. As opposed to water, the active medium forming bodies of Venusian fauna may possess retarded plasticity. These properties are typicalfor, e.g., resins. It takes a lot of time with such medium to change its shape.

Important (for life exsistance) chemical components are present on Venus, though the abundance of water vapor near the surface is insignificant, approximately $20 \mathrm{ppm}$. On the Earth, the energy $h v$ required to form a conventional organic molecule $\left(\mathrm{CH}_{2} \mathrm{O}\right)$ is $5.1 \mathrm{eV}$. However, instead of energy corresponding to UV radiation with a wavelength of around $240 \mathrm{~nm}$, our photosynthesis mechanism uses the total energy of several photons with a wavelength of around $680 \mathrm{~nm}$ (the energy is $1.8 \mathrm{eV}$ ). Photosynthesis on Earth exploits two pigments and operates in two spectral subranges, 640-680 and 410-450 $\mathrm{nm}$ [23]; moreover, it requiresthe radiation in both subranges simultaneously. The final effect is the accumulation of energy in adenosine triphosphate (ATP). 
Photosynthesis is blocked by radiation with a wavelength shorter than $300 \mathrm{~nm}$ and substantially impeded by infrared (IR) radiation, like in greenhouses illuminated by xenon arc lamps having strong IR radiation. From comparison of the curves for Earth and Venus in Figure 6, it follows that the requirements of terrestrial-type photosynthesis for radiation near the surface of Venus are satisfied in the red and near-IR ranges, while radiation at $410-450 \mathrm{~nm}$ is absorbed by the atmosphere. For photosynthesis, the hypothetical flora of Venus may use only a doubly bounded spectral interval of 520-920 nm (1.36-2.38 eV) and an additional IR band of 980-1025 nm (1.21-1.27 eV).

The VENERA missions were intended to provide a common understanding of the physical conditions on its surface and planet's atmosphere. But the obtained results are revolutionary asa dozen of Venusian hypothetical leaving creatures are found in the TV images of VENERA missions. We need an urgent implementation of a new mission to explore the surface of Venus and to confirm existance of fauna and flora there. The mission will have to be special and much more sophisticated than the VENERA missions, though well within current technical realm.

\section{References}

[1] Keldysh M. V. (Ed.). First Panoramas of the Surface of Venus. (In Russian). Moscow, Fizmatlit, 1979. 132 p.

[2] Selivanov A. S., Chemodanov V. P., Naraeva M. K. et al., TV Experiment on the Venus Surface. Kosmich. Issled., 14, 5, 1976, pp. 674-677.

[3] Selivanov A. S., Gektin Yu. M., Gerasimov M. A., Nosov B. I., Naraeva M. K. et al. Continuation of the TV Investigation of Venus surface by means of landers. Kosmich. Issled., 21, 2, 1983. pp. 176-182.

[4] Ksanfomality L. V. Planet Venus. (In Russian). Moscow, Fizmatlit, 1985. 376 p.

[5] Baklunov A. M., Karyagin V. P., Kovtunenko V. M. et al. Automatic Interplanetary Stations VENERA-13, -14. Kosmich. Issled., 21, 2, 1983, pp. 151-153.

[6] Moshkin B. E., Economov A. P., Moroz V. I. et al., Spectrophotometric Experiment on VENERA-13, -14 Landers. Kosmich. Issled., 21, 2, 1983, pp. 236-245.

[7] Surkov Yu. A., Moskaleva L. P., Scheglov O. P. (1983) Elemental composition of rocks on Venus. Kosmich. Issled., 21, 2, 1983, pp. 308-317.

[8] von Zahn U, Kumar S, Niemann H, Prinn R. Composition of the Venus atmosphere. In: Venus, Hunten D. M., Colin L., Donahue, T. M., Moroz V. I. (Eds.) Univ Arizona Press, 1983, pp. 299-430.

[9] Lewis J. S. Physics and chemistry of the solar system. (2nd edn). Academic Press. 2004.

[10] Schaefer L., Fegley B. Heavy metal frost on Venus. Icarus, 168, 2004, pp. 215-219.

[11] Gektin M, Panfilov A. S. Statistics of contrasts and peculiarities of the relief on panoramas of the surface of Venus. Kosmich. Issled. 16, 4, 1978, pp. 557-562.

[12] Volkov V. P. Chemistry of atmosphere and surface of Venus. Nauka, Fizmatlit. 1983. 205 p.

[13] McGill G. E., Warner J. L., Malin M. C. Topography, surface properties and tectonic evolution. In: Venus. Hunten D. M., Colin L., Donahue T. M., Moroz V. I. (Eds). The Univ of Arizona Press, 1983, pp. 69-136.

[14] Khodakovsky I. L. Atmosphere-surface interaction on Venus and implications for atmospheric evolution. Planet Space Sci., 8, 1982, pp. 803-817.

[15] Brackett R. A., Fegley B., Arvidson R. E. Volatile transport on Venus and implications for surface geochemistry and geology. J. Geophys. Res., 100, 1995, pp. 1553-1563.

[16] Ksanfomality L. V. Possible detection of life on the planet Venus. Doklady Physics, 57, 9, 2012, pp. 367-372.

[17] Ksanfomality L. V. Quest for hypothetic fauna of Venus at the VENERA-14 landing site: Amisadas. Doklady Physics 58, 3, 2013, pp. 272-276.

[18] Ksanfomality L. V. Hypothetical flora and fauna of Venus. Acta Astronautica 105, 2014, 521-533.

[19] Ksanfomality L. V. Possible Signs of Life on the Planet Venus. International Journal of Astronomy and Astrophysics (IJAA), 3, pp. 57-79, 2013.

[20] L. V. Ksanfomality. Looking for Mobility of Hypothetical Venusian Fauna // Advances in Zoology and Botany 1, 2, 2013, pp. 25-29.

[21] Ksanfomality L. V., Goroshkova N. V. and Khondirev V. K. Wind Velocity at the Venus Surface Obtained by Means of Acoustic Measurements, Kosmich. Issled., 21, 2, 1983, pp. 218-224.

[22] Florensky, K. P., Basilevsky, A. T., Kruchkov, V. P., et al. Geological-Geomorphological Analysis of the VENERA-13 and VENERA-14 Panoramas. Kosmich. Issled., 21, 3, 1983, pp. 340-350.

[23] Blankenship, R. E., Molecular Mechanisms of Photosynthesis, New York: Wiley, 2008. 